\title{
IMPROVING THE PERFORMANCE AND REDUCING BIT ERROR RATE ON WIRELESS DEEP FADING ENVIRONMENT RECEIVERS
}

\author{
${ }^{1}$ K. Jayanthi, ${ }^{2}$ V.P. Arunachalam and ${ }^{3}$ C. Vasanthanayaki \\ ${ }^{1,3}$ Department of Electronics and Communication Engineering, Coimbatore, Tamil Nadu, India \\ ${ }^{1,2}$ SNS College of Technology, Coimbatore, Tamil Nadu, India \\ ${ }^{3}$ Government College of Technology, Coimbatore, Tamil Nadu, India
}

Received 2013-10-04; Revised 2013-11-08; Accepted 2013-11-29

\begin{abstract}
One of the major challenges in wireless communication system is increasing complexity and reducing performance in detecting the received digital information in indoor and outdoor Environments. Consequently to overcome this problem we analyze the delay performance of a multiuser with perfect channel state information transmitting data on deep fading environment. In this proposed system, the Wireless Deep Fading Environment (WDFE) creation for causing a Nakagami Multipath Fading Channel of fading figure ' $\mathrm{m}$ ' is used to rectify the delay performance over the existing Rayleigh fading channel. In this WDFE receivers received coherent, synchronized, secured and improved signal strength of information using a Multiuser Coherent Joint Diversity (MCJD) with Multi Carrier-Code Division Multiple Access (MC-CDMA). The MCJD in ' $\mathrm{M}$ ' branch of antennas are used to reduce the Bit Error Rate (BER) and MC-CDMA method is used to improve the performance. Therefore, in this proposed system we accompany with MCJD and MC-CDMA is very good transceiver for next generation wireless system of an existing $3 \mathrm{G}$ wireless system. Overall, this experimental results show improved performance in different multiuser wireless systems under different multipath fading conditions.
\end{abstract}

Keywords: Delay Performance, Multiuser Coherent Joint Diversity, Nakagami Multipath Fading Channel, Diversity Gain

\section{INTRODUCTION}

In wireless communication a system, a transmitted signal is subjected to scatter on different kind of obstacles in deep fading environments and the receivers receive many signals with differed phase and amplitude values. All signals superpose at the receiving antenna and cause multipath fading (Tse and Viswanath, 2005). Different multipath fading models are used to describe the behavior of Wireless Deep Fading Environment (WDFE) in indoor and outdoor environments (Alsaadi and Elmirghani, 2009). Additive White Gaussian Noise (AWGN), Rayleigh fading channel, Rician fading channel and Nakagami Multipath Fading Channel (NMFC) are some of the recent multipath fading models used in communication arena. The Rayleigh fading channel uses multipath Non Line Of Sight (NLOS) with fading figure of $\mathrm{m} \leq 0.5$. In the Rician fading channel, only the Line Of Sight (LOS) and single path of NLOS are used with fading figure of $\mathrm{m}=1$ (Gill and Khanna, 2011). In the NMFC, both LOS and NLOS multipath signals are considered with fading figure $(\mathrm{m})$ value of 1 to $\infty$, because of which this model is more suitable for both indoor and outdoor environments in WDEF. Figure 1a represents the problem of WDFE in indoor and outdoor Environments. Presences of various kinds of Corresponding Author: K. Jayanthi, Department of Electronics and Communication Engineering, Coimbatore, Tamil Nadu, India 
man-made and natural obstacles do not allow the transmitted waves to directly reach the receiving antenna. The obstacles block the emitted electromagnetic waves. The waves coming from all directions due to reflection, diffraction and scattering caused by obstacles are received and cause superimposition. This kind of problem imposed by multipath effects is a delay spread, in which multiple copies of a signal arriving at different times spread out each data symbol in time and it degrades the performance of a wireless system (Yang et al., 2012). In this proposed method considered two fading cases. In the first case, observed the indoor multipath deep fading problem in multi-story building. We received the signals from different floors at different space using Wi-Fi connectivity and broadband wireless data card receiver device. In the second case, observed the signal reception from outdoor multipath fading problem in a forest area with more trees.

In, both cases, the increased distance from base station to receiver degrades the received signal strength, since we are not able to receive the particular channel reception. This problem is shown in Fig. 1b, for receiving antenna notable to receive the LOS and NLOS signals. NMFC is used to consider the effect of imperfect channel fading estimation, which is a critical issue in wireless system development (Duong et al., 2012). In this study, a system is proposed using Multiuser Coherent Joint Diversity (MCJD) with Multi Carrier Code Division Multiple Access (MC-CDMA) to overcome the problem of multipath fading.

The MCJD is used to obtain negligible bit error rate (Chen and Ling, 2010). The coherent diversity method of MRC Space diversity (MRC-SD) with polarization, Time diversity (TD) and Frequency diversity (FD) are combined to design the Multiuser Coherent Joint Diversity (MCJD) (Narasimhamurthy et al., 2012). The goal of these techniques is to achieve the full range of diversity, multiplexing and directivity tradeoffs inherent to multiuser systems (Ban et al., 2009). The MC-CDMA is used to improve the performance of next generation wireless system. MC-CDMA receivers employ all the received signal energy scattered in the frequency domain (Giannetti et al., 2008). It is based on a combination of the Code Division Multiple Access (CDMA) scheme and Orthogonal Frequency Division Multiplexing (OFDM) signaling. MC-CDMA transmitter spreads the original signal using a given spreading code in the frequency domain (Glisic, 2004). Fraction of the symbol is converted into chip of the spreading code and transmitted through a different subcarrier which is used to design a secured wireless system. Good performance and the flexibility to accommodate wireless traffic are incorporated in MC-CDMA, compared with CDMA and OFDM (Ling and Milstein, 2009). In the existing systems performance of transmission modes are evaluated by calculating the probability of high Bit Error Rate (BER) versus the Signal Noise Ratio (SNR). In section-2 describes MCJD experimental process and statistical analysis of the proposed receiver. Section-3 summarizes the simulation results and discussion. Finally, section-4 concludes the work of proposed system over existing system.

\section{PROPOSED EXPERIMENTAL METHOD}

A proposed experimental block diagram is shown in Fig. 2. The MC-CDMA transceiver which is implemented by selecting sets of communication channels and determining the channel numbers with avoiding interference and multi-path fading (Seo, 2012). The work considered whether the selected channels are a list of good channels can be generated. For this transmitter system at this instant a Successive Interference Cancellation (SIC) is planned the multicarrier synchronous CDMA system, where in the output of a convolutional encoder modulates band limited spreading waveforms at different subcarrier frequencies. This resultant transmitting signal is transmitted over the NMFC (Lodhi et al., 2008). There are many radio channels in which fading in encountered that are basically LOS communication links with NLOS multipath components arising from secondary reflection, or signal paths, from surrounding terrain (Meyr et al., 1997). In every subband, the SIC receiver successively detects signals.

The SIC receiver employs Multiuser Coherent Joint Diversity combining (MCJD) for detection of the desired user and feeds a soft decision decoder. At last, we analyzed the impact of channel uncertainty on the performance of this proposed system which is used to synchronize the system and improved the output performance (Radaydeh, 2007). The MCJD diversity with MC-CDMA is used to maintain consistent received signal strength. 


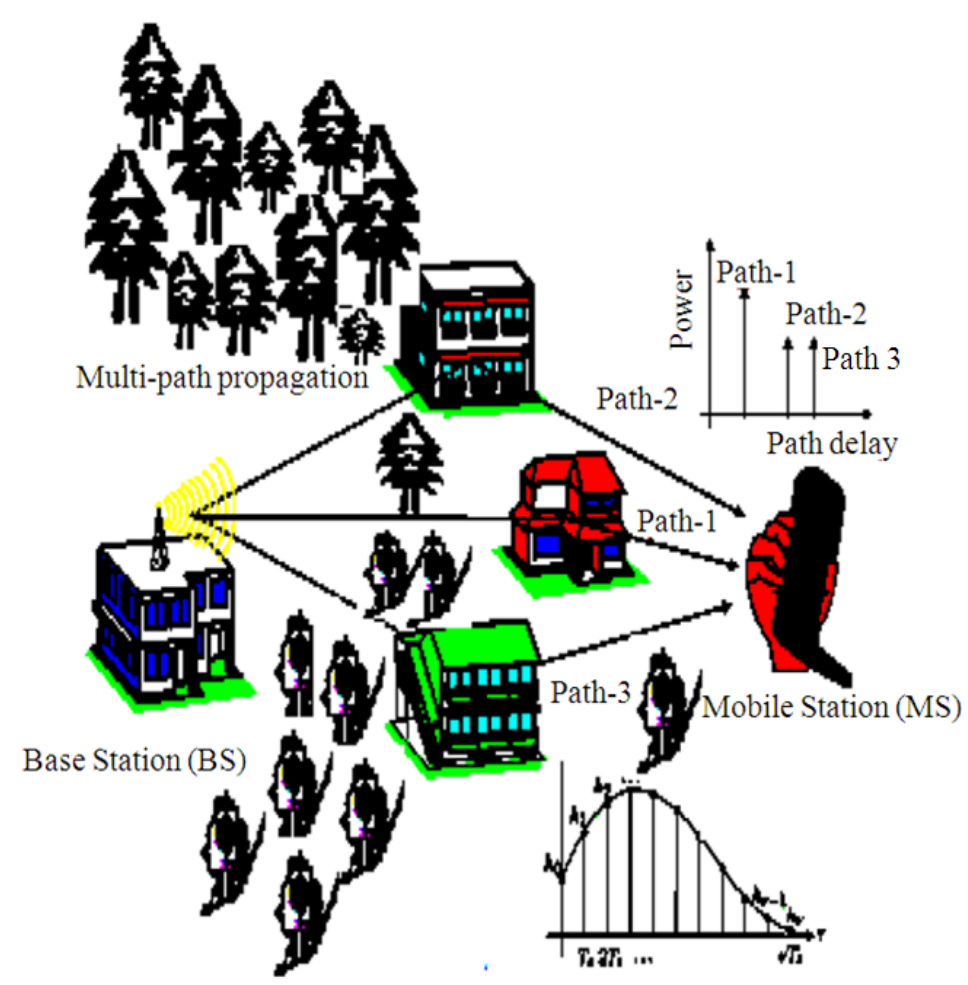

(a)

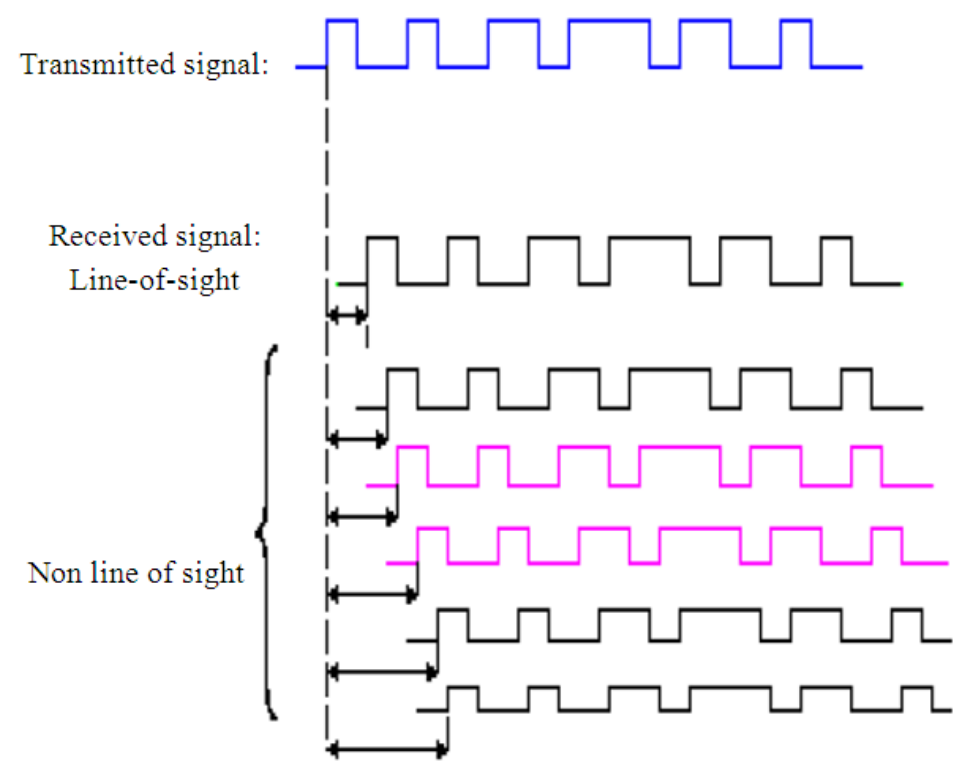

Delays

(b)

Fig. 1. (a) Representation of problem on wireless deep fading environment (b) Representation of signal transmitted and reception of WDFE condition 


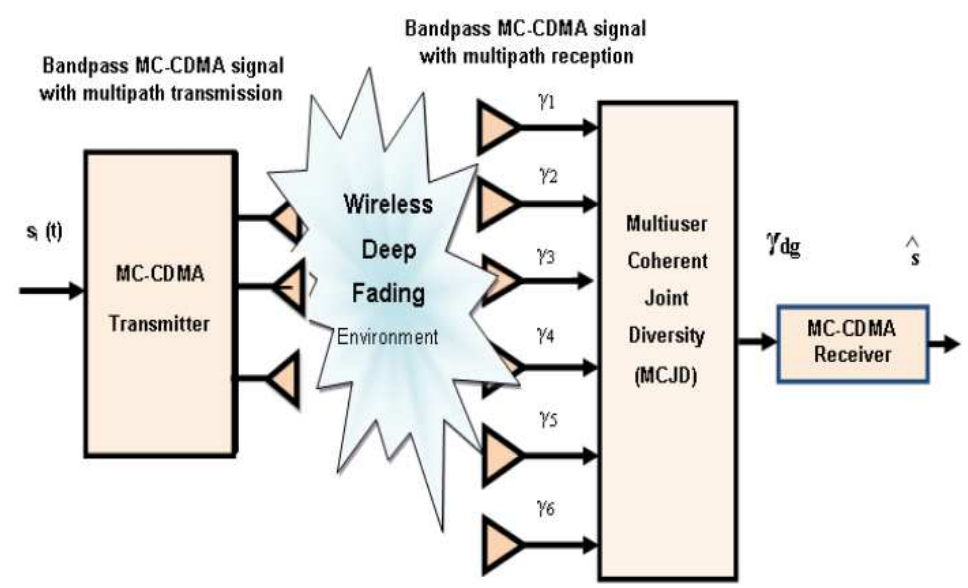

Fig. 2. Proposed experimental wireless system block diagram

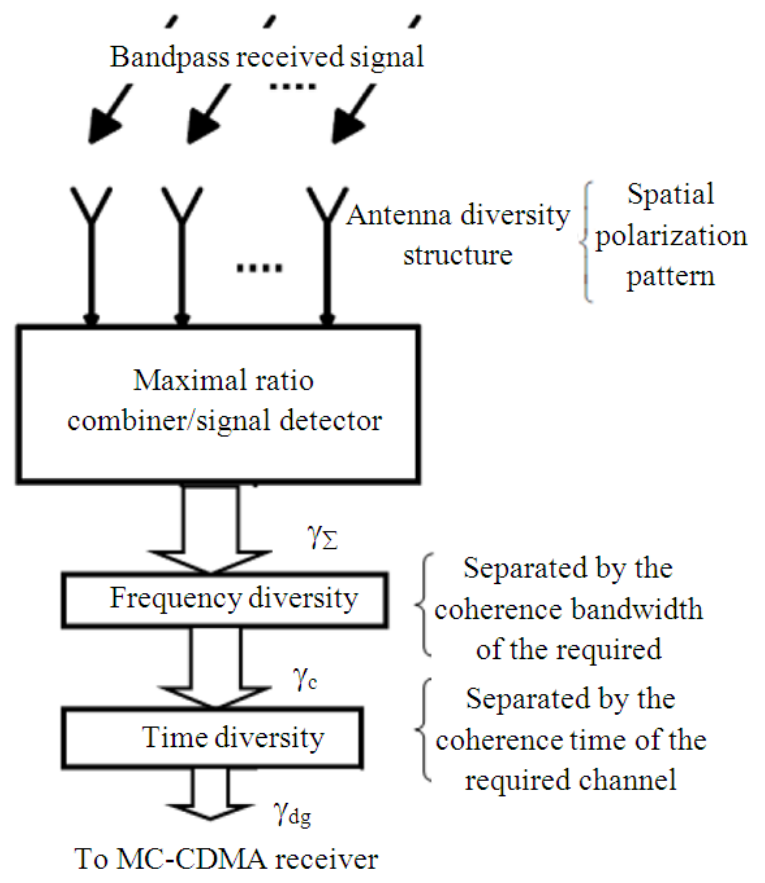

Fig. 3. Experimental process of proposed MCJD combiner

\subsection{MCJD Experimental Process}

A Proposed MCJD Combiner system is shown in Fig. 3. The receiver side, six antennas are used to cover the $360^{\circ}$ propagation phase reception and each antenna maintains the phase difference of $60^{\circ}$. Since using polarization diversity, the signal polarization is subjected to change due to diffraction, refraction and reflection (Alsaadi and Elmirghani, 2009). In this work both combination of Right Hand Circular Polarization (RHCP)-Left Hand Circular
Polarization (LHCP) and Vertical-Horizontal antennas are examined. However, the components of received signals in the orthogonal antennas adequately discriminated to achieve a sufficiently low correlation coefficient between the received signals. Resultant of the MRC diversity is sum of six antenna polarization of an unequal energy signal (Coskun and Kucur, 2012). In MRC output $\gamma_{\Sigma}$ is a weighted sum of all polarization diversity branches. In frequency diversity the information is transmitted on $\mathrm{L}$ different carrier frequencies. The coherence bandwidth $(\Delta f)_{c}$ is defined as the range of frequencies over which two frequency components have a strong amplitude correlation. In this diversity the information is transmitted L times, this output is SNR of required channel $\gamma_{c}$. Time diversity, the separations between the successive time slots must equal or exceed the coherence time $(\Delta t)_{c}$ of the channel. The coherence time $(\Delta t)_{c}$ is defined as the time duration over which two received signals have a strong amplitude correlation, yield is diversity gain $\gamma_{\mathrm{dg}}$ or $\gamma_{\text {MCID-dg. }}$. Accordingly, for this process we used MC-CDMA receiver is used to receive the required channel information.

\subsection{The Statistical Analysis of MCJD Combiner}

In this section, we derived the statistical analysis of MCJD combiner. These expressions can be applied directly for computing the Bit Error Rate (BER) for different receiver schemes. The MCJD Combiner functional diagram is shown in Fig. 4. The MRC select $M$ signals from $\mathrm{k}$ received signals having sum of SNR (Tan et al., 2011). The differential detector outputs, which are all co-phased, are then combined by a MRC. For $\mathrm{M}=1$, there is no diversity and if $\mathrm{M}=\mathrm{k}$, all the received signals $\mathrm{L}$ are combined and MRC is considered (Lee et al., 2012). 


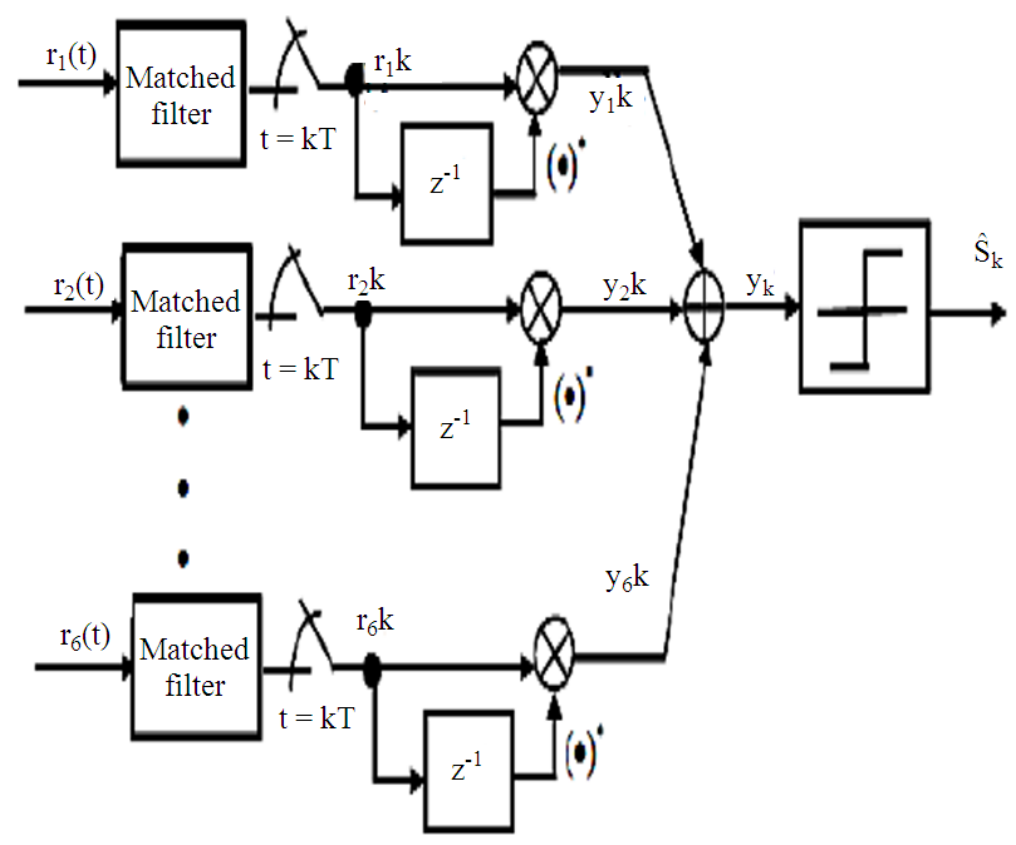

Fig. 4. Functional diagram of MCJD combiner using $M=k=6$

From Fig. 2, the MC-CDMA transmitted signal is Equation (1):

$s(t)=V(t) \cos \left(2 \pi f_{c} t+\theta\right)$

The received complex envelop in each MRC Diversity branch is Equation (2):

$r_{m(t)}=g_{m}(t) s(t)+n_{m}(t)$

Where:

$\mathrm{s}(\mathrm{t})=$ The complex envelop of the transmitted signal $g_{m}(t)=$ The complex gain in the $m^{\text {th }}$ Gaussian channel $\mathrm{n}_{\mathrm{m}}(\mathrm{t})=$ The $\mathrm{m}^{\mathrm{th}}$ WDFE/NMFC noise

All the $g_{m}(t)$ and $n_{m}(t)$ are statistically independent. The coherent diversity of linear demodulation with the receiver structure is shown in Fig. 4. The output of the matched filters at, $t=k T, r_{m, k}$ $=\mathrm{g}_{\mathrm{m}, \mathrm{k}} \mathrm{s}_{\mathrm{k}}+\mathrm{n}_{\mathrm{m}, \mathrm{k}}, \mathrm{m}=1,2, \ldots \mathrm{N} . \mathrm{s}_{\mathrm{k}}$ is the $\mathrm{k}^{\text {th }}$ transmitted symbol, for this system model we used $\mathrm{N}=6$. The transmitted energy per bit is $\mathrm{E}_{\mathrm{s}}$ and the received signal is different from $E_{s}$ which stands for the average energy/bit that enters MRC diversity channel. In frequency and time diversity systems, $E_{s}=E_{b} / N$, because the actual transmitted energy $E_{b}$ must be split equally among $\mathrm{N}$ diversity channels.

\subsection{Estimation of MCJD Probability BER}

The received SNR per branch is Equation (3):

$\Gamma_{8, \mathrm{MC}-\mathrm{CDMA}}=\frac{\sigma_{\mathrm{g}}^{2}}{\mathrm{~N}_{0}}=\frac{\mathrm{E}_{8}}{\mathrm{~N}_{0}}=\left(\frac{1}{\mathrm{~N}_{0}}\right) \frac{\mathrm{E}_{\mathrm{b}}}{\mathrm{N}_{0}}$

Where:

$\sigma_{\mathrm{g}}=$ The diversity gain of zero mean complex Gaussian random variable

$\mathrm{N}_{0}=$ The WDFE noise power spectral density.

The decision variable at the combined output is Equation (4):

$$
\begin{aligned}
\mathrm{y}_{\mathrm{k}}= & \sum_{\mathrm{m}=1}^{\mathrm{N}} \mathrm{r}_{\mathrm{m}, \mathrm{k}} \mathrm{r}_{\mathrm{m}, \mathrm{k}-1}^{*}=\sum_{\mathrm{m}=1}^{\mathrm{N}}\left(\mathrm{g}_{\mathrm{m}, \mathrm{k}} \mathrm{s}_{\mathrm{k}}+\mathrm{n}_{\mathrm{m}, \mathrm{k}}\right) \\
& \left(\mathrm{g}_{\mathrm{m}, \mathrm{k}-1} \mathrm{~S}_{\mathrm{k}-1}+\mathrm{n}_{\mathrm{m}, \mathrm{k}-1}\right)^{*}
\end{aligned}
$$

If $\operatorname{Re}\left(y_{k}\right)$ is greater than zero, the receiver decides that the $\mathrm{k}^{\text {th }}$ information bit is $\mathrm{s}_{\mathrm{k}}=1$, else it decides that $\mathrm{s}_{\mathrm{k}}$ $=-1$. As in the past, we set $s_{\mathrm{k}-1}=1$ and $\mathrm{s}_{\mathrm{k}}=1$ in the BER analysis. The information bit is $s_{k}=1$ and the decision variable $\mathrm{y}_{\mathrm{k}}$ becomes where Equation (5):

$$
\mathrm{y}_{\mathrm{k}}=\sum_{\mathrm{m}=1}^{\mathrm{N}}\left(\mathrm{g}_{\mathrm{m}, \mathrm{k}}+\mathrm{n}_{\mathrm{m}, \mathrm{k}}\right)\left(\mathrm{g}_{\mathrm{m}, \mathrm{k}-1}+\mathrm{n}_{\mathrm{m}, \mathrm{k}-1}\right)^{*}=\sum_{\mathrm{m}=1}^{\mathrm{N}} \mathrm{x}_{\mathrm{m}} \mathrm{y}_{\mathrm{m}}^{*}
$$


Where:

$$
\begin{gathered}
\mathrm{X}_{\mathrm{m}}=\mathrm{g}_{\mathrm{m}, \mathrm{k}}+\mathrm{n}_{\mathrm{m}, \mathrm{k}} \\
\mathrm{Y}_{\mathrm{m}}=\mathrm{g}_{\mathrm{m}, \mathrm{k}-1}+\mathrm{n}_{\mathrm{m}, \mathrm{k}-1}
\end{gathered}
$$

The noise term $\mathrm{n}=\sum_{\mathrm{m}=1}^{\mathrm{N}} \mathrm{g}_{\mathrm{m}, \mathrm{k}}^{*} \mathrm{n}_{\mathrm{m}, \mathrm{k}}$ and the fading gains $\mathrm{g}_{\mathrm{m}, \mathrm{k}} \mathrm{s}_{\mathrm{k}}$ 's, $\mathrm{n}$ is a zero mean complex Gaussian random variable with a variance of Equation (6):

$$
\begin{aligned}
\sigma_{n}^{2} & =\frac{1}{2} E\left[\left|n^{2}\right|\right]=\frac{1}{2} E\left[\left(\sum_{m=1}^{N} g_{m, k}^{*} n_{m, k}\right)\left(\sum_{j=1}^{N} g_{j, k} n_{j, k}^{*}\right)\right] \\
& =\frac{1}{2} E\left[\sum_{m=1}^{N} \sum_{j=1}^{N} g_{m, k}^{*} g_{j, k} n_{m, k}, k^{n^{*}}\right], k \\
& =N_{0}\left(\sum_{m=1}^{N}\left|g_{m, k}^{2}\right|\right)=N_{0} z
\end{aligned}
$$

In the case of a MRC diversity, the overall signal $\mathrm{z}$ is:

$$
\begin{aligned}
& \mathrm{Z}=\sum_{\mathrm{m}=1}^{\mathrm{N}}\left|\mathrm{g}_{\mathrm{m}, \mathrm{k}}\right|^{2}=\sum_{\mathrm{m}=1}^{\mathrm{N}} \mathrm{Z}_{\mathrm{m}} \\
& \mathrm{Z}_{\mathrm{m}}=\left|\mathrm{g}_{\mathrm{m}, \mathrm{k}}\right|^{2}
\end{aligned}
$$

The decision statistics are weighted to form an overall decision statistic as shown in Fig. 4. The outputs of the correlates are denoted as $\mathrm{z}^{-1}$. They are weighted by $\alpha_{1}, \alpha_{2} \ldots \ldots \alpha_{\mathrm{M}}$. The weighting coefficients are based on the SNR from each correlator output. If the SNR was small out of a particular correlated. It is assigned a small weighting factor. The results of the set of linear equation $\mathrm{Rm}$ of quadratic form of the zero mean complex Gaussian random variables $\mathrm{X}_{\mathrm{m}}$ and $\mathrm{Y}_{\mathrm{m}}$ is Equation (7):

$$
\begin{aligned}
& R_{\mathrm{m}}=\frac{1}{2} \mathrm{E}\left[\left(\begin{array}{c}
\mathrm{X}_{\mathrm{m}} \\
\mathrm{Y}_{\mathrm{m}}
\end{array}\right)\left(\mathrm{X}_{\mathrm{m}}^{*} \mathrm{Y}_{\mathrm{m}}^{*}\right)\right] \\
& =\frac{1}{2} \mathrm{E}\left[\left(\begin{array}{c}
\mathrm{g}_{\mathrm{m}, \mathrm{k}}+\mathrm{n}_{\mathrm{m}, \mathrm{k}} \\
\mathrm{g}_{\mathrm{m}, \mathrm{k}-\mathrm{l}}+\mathrm{n}_{\mathrm{m}, \mathrm{k}-\mathrm{l}}
\end{array}\right)\left(\mathrm{g}_{\mathrm{m}, \mathrm{k}}^{*}+\mathrm{n}_{\mathrm{m}, \mathrm{k}}^{*} \mathrm{~g}_{\mathrm{m}, \mathrm{k}}^{*}+\mathrm{n}_{\mathrm{m}, \mathrm{k}-1}^{*}\right)\right] \\
& =\left[\begin{array}{cc}
\sigma_{\mathrm{g}}^{2}+\mathrm{N}_{0} & \sigma_{\mathrm{g}}^{2} \mathrm{~J} \\
\sigma_{\mathrm{g}}^{2} \mathrm{~J} & \sigma_{\mathrm{g}}^{2}+\mathrm{N}_{0}
\end{array}\right]
\end{aligned}
$$

where, $\mathrm{J}=\mathrm{J}_{0}\left(2 \pi \mathrm{f}_{\mathrm{d}} \mathrm{T}\right), \mathrm{J}_{0}\left(2 \pi \mathrm{f}_{\mathrm{d}} \mathrm{T}\right)$ is the zero-order Bessel function, $f_{d}$ is the maximum Doppler frequency and $1 / T$ is the bit rate. The characteristic function approach is used to determine the BER. The multichannel wireless communication transceiver, transmitting information over WDFE, the decision variable at the detector can be represented in the quadratic form:

$$
\begin{aligned}
\mathrm{D} & =2 \operatorname{Re}\left(\mathrm{Y}_{\mathrm{k}}\right) \\
& =\sum_{\mathrm{m}=1}^{\mathrm{N}}\left(\mathrm{X}_{\mathrm{m}} \mathrm{Y}_{\mathrm{m}}^{*}+\mathrm{X}_{\mathrm{m}}^{*} \mathrm{Y}_{\mathrm{m}}\right) \\
& =\sum_{\mathrm{m}=1}^{\mathrm{N}} \mathrm{D}_{\mathrm{m}}
\end{aligned}
$$

where, $D_{m}$ is a diagonal matrix with diagonal elements of $\mathrm{X}_{\mathrm{m}}$ and $\mathrm{Y}_{\mathrm{m}}$, the expression is:

$$
\begin{aligned}
D_{m} & =X_{m} Y_{m}^{*}+X_{m}^{*} Y_{m} \\
& =\left[\begin{array}{ll}
X_{m}^{*} & Y_{m}^{*}
\end{array}\right]\left[\begin{array}{ll}
0 & 1 \\
1 & 0
\end{array}\right]\left[\begin{array}{c}
X_{m} \\
Y_{m}
\end{array}\right]
\end{aligned}
$$

In the NMF channel used, the $\mathrm{L}$ pairs $\left\{\mathrm{X}_{\mathrm{m}}, \mathrm{Y}_{\mathrm{m}}\right\}$ are mutually statistically independent and identically distributed (i.i.d).

The probability of BER $\mathrm{P}_{\mathrm{b}}{ }^{\mathrm{MCJD}}$ is:

$$
\begin{aligned}
\mathrm{P}_{\mathrm{b}}{ }^{\text {MCJD }} & =\mathrm{p}[\mathrm{D}<0] \\
& =\left[\frac{1}{2}\left(1-\frac{\mathrm{J}}{1+\Gamma_{\mathrm{s}}^{-1}}\right)\right]^{\mathrm{N}} \sum_{\mathrm{j}=0}^{\mathrm{N}-1}\left(\begin{array}{c}
\mathrm{n}+\mathrm{j}-1 \\
\mathrm{j}
\end{array}\right)\left[\frac{1}{2}\left(1+\frac{\mathrm{J}}{1+\Gamma_{\mathrm{s}}^{-1}}\right)\right]^{\mathrm{j}}
\end{aligned}
$$

In Equation (8), the branch SNR is $\Gamma_{\mathrm{s}}=\sigma_{\mathrm{g}}^{2} / \mathrm{N}_{0}$. For $\mathrm{k}=$ 1 is called as No Diversity. In this proposed experimental process ' $d$ ' is the diversity order of the system. The antenna branch converted the diversity order is Equation (9):

$\mathrm{d}=\mathrm{k}-1 ; \quad 1 \leq \mathrm{k} \leq 6$

The diversity order indicates the slope of the average probability of error as a function of average SNR changes with diversity. Finally, those simulation results are shown in section 4 .

\subsection{Estimation of MCJD Diversity Gain}

Diversity gain reflects that several antenna elements are in a fading dip simultaneously; the probability for very low signal levels is thus decreased by the use of multiple antenna elements. Beam forming gain reflects the combiner perform an averaging over the noise at different antennas. Thus, even if the signal levels at all antenna elements are identical; the combined output SNR is larger than the SNR at a single-antenna element. With the WDFE considered to be slowly varying relative to the symbol duration $\mathrm{T}$ in Fig. 4, estimated and then removed the unknown phase shift $\theta_{\mathrm{k}}$ at each diversity branch with sufficient, where $\mathrm{E} / \mathrm{N}_{0}$ is the symbol energy to noise spectral density ratio. The received instantaneous output SNR of the linear coherent combiner is: 


$$
\begin{aligned}
& \gamma_{i}=\frac{\left(\frac{E}{N_{0}}\right)\left|\sum_{k=1}^{d} a_{k} \alpha_{k} e^{j \theta_{k}}\right|^{2}}{d} \\
& =\frac{E}{N_{0}} \alpha_{k}^{2} \sum_{k=1}^{d}\left|a_{k}\right|^{2} ; k=1,2, \ldots ., d
\end{aligned}
$$

The requirement is to maximize $\gamma_{\mathrm{i}}$ in Equation (10) with respect to the $a_{k}$. The optimal weighting factor $a_{k}$ for the $\mathrm{k}^{\text {th }}$ diversity branch has a magnitude amplitude $\alpha_{\mathrm{k}}$ of the signal and a phase that cancels by polarization diversity, the signals phase $\theta_{\mathrm{k}}$ to within some value that is identical for all the 'd' diversity branches. If permits the fully coherent addition of the ' $d$ ' receiver outputs by the linear MRC combiner. The Equation (10) with the equality sign defines the instantaneous output $\mathrm{E} / \mathrm{N}_{0}$ of the $\mathrm{MRC}$ is:

$$
\gamma_{\mathrm{MRC}}=\gamma_{\Sigma} \sum=\sum_{\mathrm{k}=1}^{\mathrm{d}} \gamma_{\mathrm{i}}
$$

Thus, at high SNR, the diversity order of MRC is d, the number of antennas and so MRC achieves full diversity order. In frequency diversity, the MRC signal of Equation (11) is received at more different frequencies. If these frequencies are spaced apart by more than the coherence bandwidth of the channel, then their fading is approximately independent and the probability is low that the signal is in a deep fade at both frequencies simultaneously. The correlation between two frequencies can be obtained from Equation (8) by setting the numerator to unity as the signals at the two frequencies occur at the same time. Thus Equation (12):

$$
\rho=\frac{1}{1+(2 \pi)^{2} S_{\tau}^{2}\left(f_{2}-f_{1}\right)^{2}}
$$

Considering the correlation coefficient of two signals that have a temporal separation $\tau$ and a frequency separation $f_{1}-f_{2}$. The $\rho$ is a function of the spacing between the two frequencies. The receiver can sum the different frequencies to recover the original information. The SNR of required frequency channel gain is Equation (13):

$$
\gamma_{\mathrm{c}}=\frac{\left(\frac{\mathrm{E}}{\mathrm{N}_{0}}\right) \gamma_{\mathrm{MRC}}}{\rho}
$$

When frequency diversity is required, the appropriate traffic is simply switched to the backup frequency. However, for critical traffic, the expense may be justified. As the wireless propagation channel is time variant, signals that are received at different times are uncorrelated. In a NMF channel, where neither transceiver is moving, the channel state is the same at all times. In such a case, the correlation coefficient is $\rho \# 1$ for all time intervals and time diversity is used. The weighting coefficient $\mathrm{Z}^{-1}$ in Fig. 4 are normalized to the output signal power of the correlated such a way that the coefficients sum to unity. The MCJD diversity gain is:

$\gamma_{\mathrm{MCJD}-\mathrm{dg}}=\frac{\gamma_{\mathrm{c}}}{\gamma_{\mathrm{av}}}$

where, $\gamma_{\mathrm{av}}$ is the average value of $\mathrm{E} / \mathrm{N}_{0}$ over WDFE. The above Equation (14) is used to calculate the MCJD combiner diversity gain. According this calculation, this diversity gain is very low and reduces the BER compared with MRC and RAKE diversity techniques. The simulation result of this experimental process is shown in section 4 .

\subsection{MC-CDMA Signal Reception}

In this section, employing multiuser detection and decoding to improve the performance of the WDFE multiuser receiver in a MC-CDMA system are considered. Therefore, to ensure numerical stability, the subsequent is regarded as the approximated extrinsic information, deinterleaved and fed back to the channel decoder of the $n$th user. Finally, the WDFE multiuser receiver receives the strongest signal among them.

\section{SIMULATION RESULTS AND PERFORMANCE ANALYSIS}

In this section, the performance analyses of proposed system statistical results are discussed. In Fig. 5 shows the comparison of the performance of WDFE with MC-CDMA transmitted signal. In this figure, the transmitted SNR was $16 \mathrm{~dB}$. The transmitted signals are propagated in free space; due to the fading effect the signal level was increased. This increased level is the fact of fading figure propagating interference addition with transmitted information.

According to Fig. 5, the NMFC was used to collect the LOS wave and NLOS multipath propagation wave, while the SNR is increased to $28 \mathrm{~dB}$. For this analysis due to the fading propagation effect the SNR value was increased and BER values also increased from transmitter to Rician Fading Channel, Rayleigh Fading Channel and NMFC. In NMFC, the SNR is on higher side along with high BER, which is the huge problem for fading effect. 


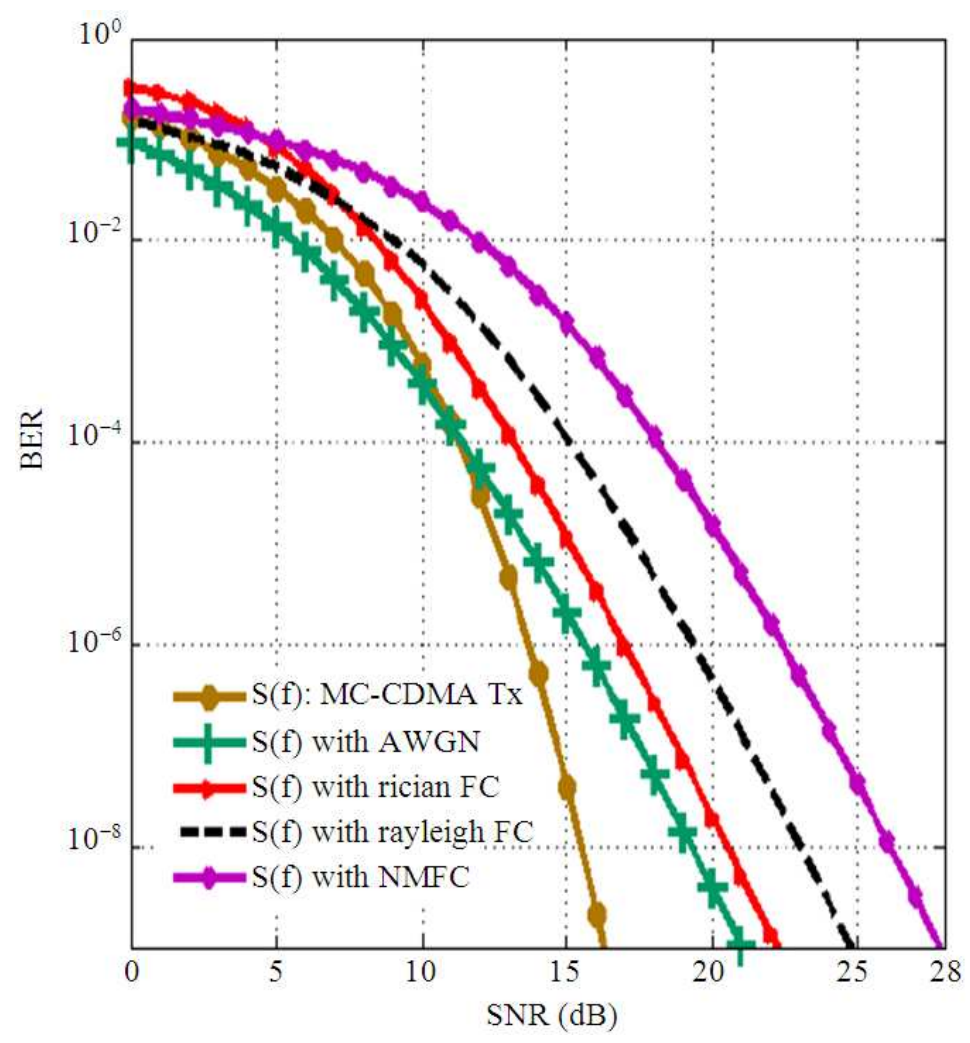

Fig. 5. Performance analysis of MC-CDMA transmitted signal with WDFE of different Fading Channel (FC)

\subsection{Performance Analysis of MCJD}

In this section, we compared the simulation results of experimental receiver performance MRC diversity $\gamma_{\text {MRC }}$ or $\gamma_{\Sigma}$ and Rake Diversity (RD) receiver of time diversity $\gamma_{\mathrm{RD}}$ form Equation (10 and 11) are used to find the SNR vs. BER with different fading propagation channel. In Fig. 6, the Rayleigh fading channel compared NMFC with both diversity receiver systems. Comparing the Fig. 5 and 6 the diversity method is used to reduce the BER value in order of $10^{-8}$ to $10^{-12}$ of the existing systems.

Equation (14) is used to reduce SNR is $9.5 \mathrm{~dB}$ shown in Fig. 7. Compare the Fig. 5 and 7; decrease the BER in the order of $10^{-8}$ to $10^{-22}$ of WDFE. In Fig. 7 achieved a BER is less than $10^{-22}$ for MCJD combiner, the obtained SNR would be enormous power saving in the maximum order of less than $10 \mathrm{~dB}$ out of $30 \mathrm{~dB}$. With this technique the SNR may decrease due to the loss of multipath components that fall outside the receive antenna beamwidth, unless the directional gain of the antenna is sufficiently large to compensate for this absent power.
At higher average SNRs the curves become less steep, leading to more of a power difference at different BER. In fact the power savings is most substantial going from no diversity to $M=k=6$ branch diversity with diminishing returns as the number of branches is increased. Thus, this system of SNR indicates that, the system requires less transmit power to achieve the good reception in reliable wireless medium.

\subsection{Performance analysis of MCJD with MC- CDMA Receiver}

The performance analyses of proposed system over OFDM, CDMA with and without diversity wireless transceiver system are compared and shown in Fig. 8.

In this Table 1, BER of the proposed system on an indoor and outdoor of WDFE are shown in which the BER is less than 10-30. In compared with other wireless transceivers, BER and SNR of the proposed system are very less. Since this proposed system has obtained negligible BER, this can be able to receive the weak signals too. 


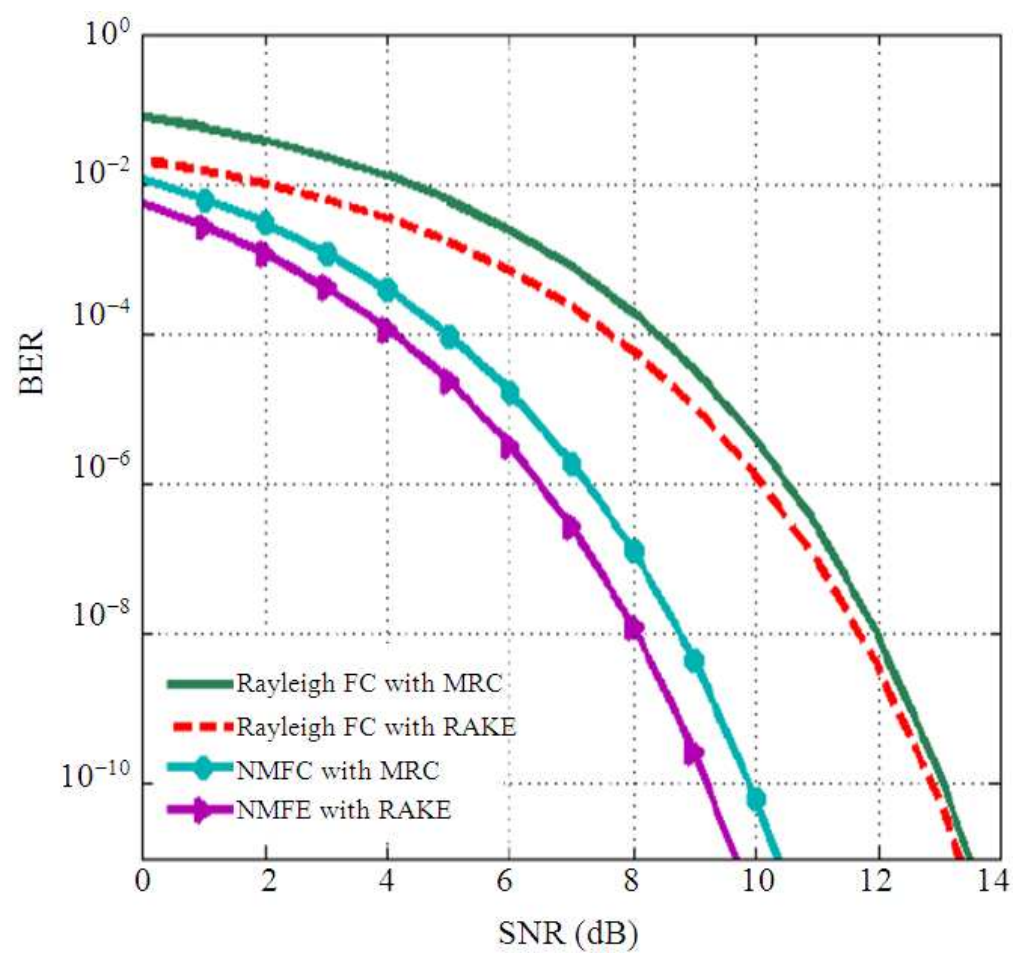

Fig. 6. Performance analysis on MRC and RAKE diversity received signal ( $\gamma_{\mathrm{MRC}}$ and $\gamma_{\mathrm{RD}}$ ) of WDFE with different Fading Channel (FC)

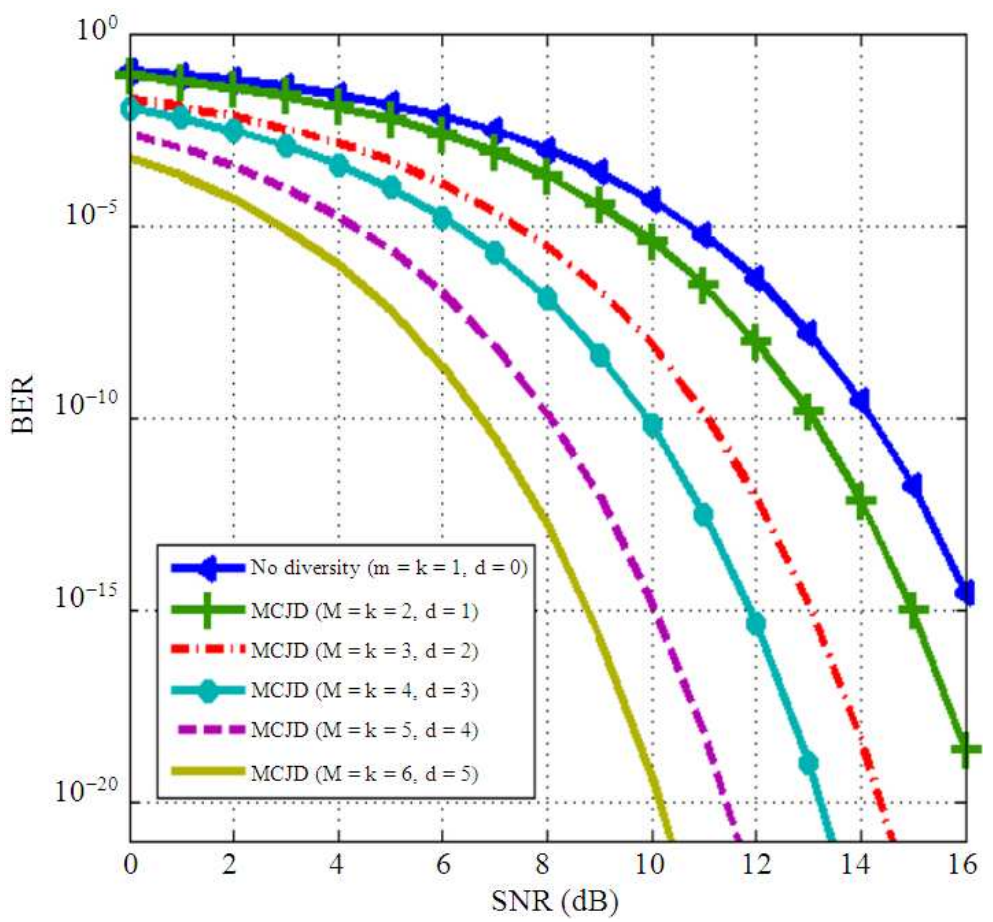

Fig. 7. Performance analysis of no diversity and MCJD combiner with diversity order $d=1,2,3,4,5$ 


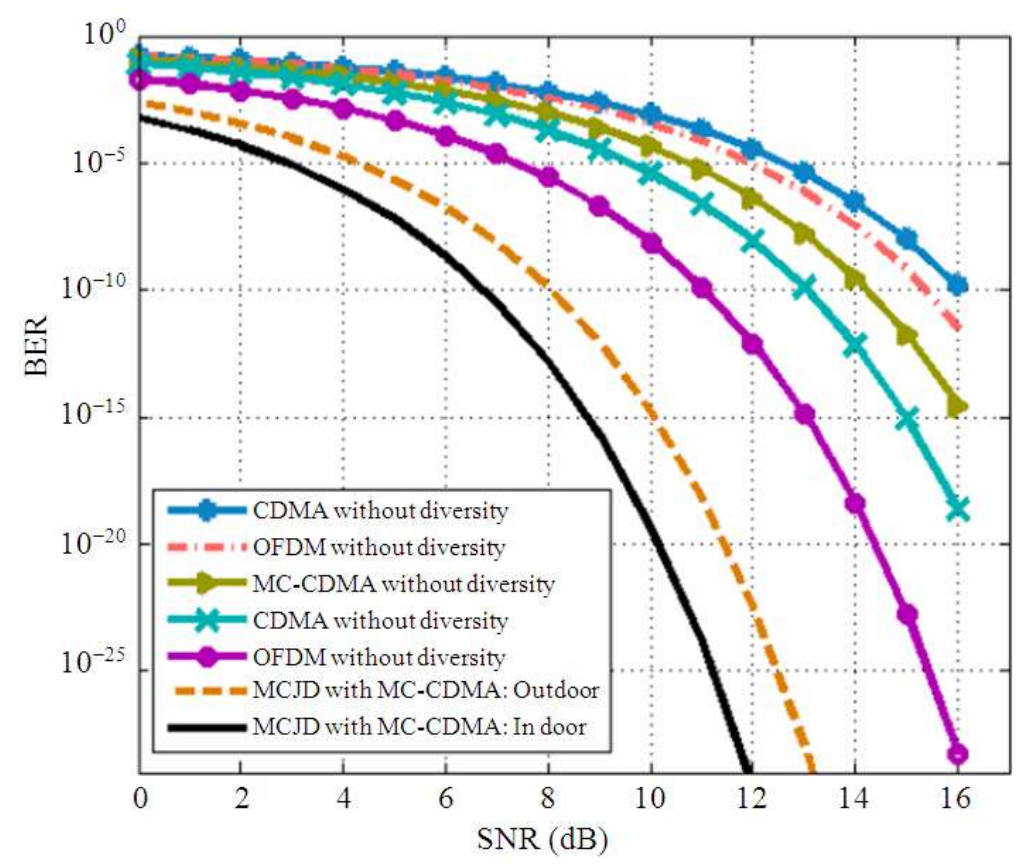

Fig. 8. Performance analysis of MCJD with MC-CDMA receiver on indoor and outdoor of WDFE

Table 1. Comparison of MCJD with MC-CDMA over CDMA/ OFDM transceiver

\begin{tabular}{lll}
\hline Wireless transceiver & SNR & BER \\
\hline MC-CDMA transmitter & 16 & $\approx 10-10$ \\
CDMA without diversity & 16 & $10-10$ \\
OFDM without diversity & 16 & $10-12$ \\
MC-CDMA without diversity & 16 & $10-15$ \\
CDMA with diversity & 16 & $10-19$ \\
OFDM with diversity & 16 & $10-29$ \\
MCJD with MC-CDMA: Out-door environment & 13 & $\leq 10-30$ \\
MCJD with MC-CDMA: Indoor environment & 12 & $\leq 10-30$ \\
\hline
\end{tabular}

\section{CONCLUSION}

In this experimental work, we proposed a multiuser coherent joint diversity combiner scheme with multicarrier code division multiple access transceivers through the wireless deep fading environment is characterized as Nakagami multipath fading channel and evaluated its performance. Using probability density function formula of the transceiver is used to obtain BER in order of less than 10-30 and observed fading channel propagation, also obtained low receiver diversity gain. Simulation results are shown for verification; we also compared the performance of without diversity, with MCJD method and MC-CDMA are used to receive the Successive Interference Cancellation and secured multiuser information's are received on WDFE receiver. Therefore, this method provides improved indoor and outdoor receiver channel quality. With these considerations, the proposed method is giving better performance compared with existing systems. Hence, this proposed system will be suitable for Fourth Generation wireless systems.

\section{REFERENCES}

Alsaadi, F.E. and J.M.H. Elmirghani, 2009. Adaptive mobile spot diffusing angle diversity MC-CDMA optical wireless system in a real indoor environment. IEEE Trans. Wireless Commun., 8: 2187-2192. DOI: 10.1109/TWC.2009.070857

Ban, T.W., W. Choi, B.C. Jung and D.K. Sung, 2009. Multi-user diversity in a spectrum sharing system. IEEE Trans. Wireless Commun., 8: 102-106. DOI: 10.1109/T-WC.2009.080326 
Chen, Y. and C. Ling, 2010. Effect of correlated nakagami-m fading on the $\varepsilon$-outage channel capacity of the decentralized two-relay network. IEEE Trans. Wireless Commun., 9: 3607-3612. DOI: 10.1109/TWC.2010.101810.100179

Coskun, A.F. and O. Kucur, 2012. Performance analysis of maximal-ratio transmission/receive antenna selection in nakagami-m fading channels with channel estimation errors and feedback delay. IEEE Trans. Veh. Technol., 61: 1009-1108. DOI: 10.1109/TVT.2012.2183650

Duong, T.Q., D.B. Da Costa, T.A. Tsiftsis, C. Zhong and A. Nallanathan, 2012. Outage and diversity of cognitive relaying systems under spectrum sharing environments in nakagami-m fading. IEEE Commun. Lett., 16: 2075-2078. DOI: 10.1109/LCOMM.2012.100812.121859

Giannetti, F., V. Lottici and I. Stupia, 2008. Iterative multi-user data predistortion for MC-CDMA communications. IEEE Trans. Wireless Commun., 7: 3823-3833. DOI: 10.1109/T-WC.2008.070382

Gill, Y.S. and R. Khanna, 2011. Analysis of data transmission techniques in rayleigh, rician and nakagami fading model under various modulation schemes. Int. J. VLSI Signal Process. Applic., 1: 108-111.

Glisic, S.G., 2004. Advanced Wireless Communications: 4G Technologies. 1st Edn., Wiley, ISBN-10: 0470867760, pp: 857.

Lee, K., N. Lee and I. Lee, 2012. Achievable degrees of freedom on k-user $\mathrm{Y}$ channels. IEEE Trans. Wireless Commun., 11: 1210-1219. DOI: 10.1109/TWC.2012.012712.111194

Ling, A. and L.B. Milstein, 2009. The effects of spatial diversity and imperfect channel estimation on wideband MC-DS-CDMA and MC-CDMA. IEEE Trans. Commun., 57: 2988-3000. DOI: 10.1109/TCOMM.2009.10.080185
Lodhi, A., F. Said, M. Dohler and A.H. Aghvami, 2008. Closed-form symbol error probabilities of STBC and CDD MC-CDMA with frequency-correlated subcarriers over nakagami-m fading channels. IEEE Trans. Veh. Technol., 57: 962-973. DOI: 10.1109/TVT.2007.906355

Meyr, H., M. Moeneclaey and S.A. Fechtel, 1997. Digital Communication Receivers, Synchronization, Channel Estimation and Signal Processing. 1st Edn., Wiley, New York, ISBN-10: 0471502758, pp: 864.

Narasimhamurthy, A.B., C. Tepedelenlioglu and Y. Zhang, 2012. Multi-user diversity with random number of users. IEEE Trans. Wireless Commun., 11: 60-64. DOI: 10.1109/TWC.2011.121911.111594

Radaydeh, R.M., 2007. Simple tight upper bounds for average SEP and BEP of coherent diversity m-ary biorthogonal signals over fading channels. IEEE Trans. Vehicular Technol., 56: 962-973. DOI: 10.1109/TVT.2007.899954

Seo, B., 2012. SINR lower bound based multiuser detector for uplink MC-CDMA systems with residual frequency offset. IEEE Commun. Lett., 16: 1612-1615.

DOI: 10.1109/LCOMM.2012.090312.121158

Tan, B.S., K.H. Li and K.C. Teh, 2011. Performance analysis of LDPC codes with maximum-ratio combining cascaded with selection combining over nakagami-m fading. IEEE Trans. Wireless Commun., 10: 1886-1894. DOI: 10.1109/TWC.2011.040511.101226

Tse, D. and P. Viswanath, 2005. Fundamentals of Wireless Communication. 1st Edn., Cambridge University Press, Cambridge, ISBN-10: 0521845270, pp: 564.

Yang, N., M. Elkashlan, P.L. Yeoh and J. Yuan, 2012. Multiuser MIMO relay networks in nakagami-m fading channels. IEEE Trans. Commun., 60: 32983310. DOI: 10.1109/TCOMM.2012.081412.110463 